\title{
Neutron capture cross sections of $\mathrm{Kr}$
}

\author{
Stefan Fiebiger ${ }^{1, \star}$, Bayarbadrakh Baramsai ${ }^{2}$, Aaron Couture $^{2}$, Milan Krtička $^{3}$, Shea Mosby ${ }^{2}$, \\ René Reifarth ${ }^{1}$, John O'Donnell ${ }^{2}$, Gencho Rusev², John Ullmann², Mario Weigand'1, and \\ Clemens Wolf ${ }^{1}$ \\ ${ }^{1}$ Goethe University Frankfurt, Germany \\ ${ }^{2}$ Los Alamos National Laboratory, USA \\ ${ }^{3}$ Charles University, Czech Republic
}

\begin{abstract}
Neutron capture and $\beta^{-}$-decay are competing branches of the s-process nucleosynthesis path at ${ }^{85} \mathrm{Kr}$ [1], which makes it an important branching point. The knowledge of its neutron capture cross section is therefore essential to constrain stellar models of nucleosynthesis. Despite its importance for different fields, no direct measurement of the cross section of ${ }^{85} \mathrm{Kr}$ in the keV-regime has been performed. The currently reported uncertainties are still in the order of 50\% [2,3]. Neutron capture cross section measurements on a $4 \%$ enriched ${ }^{85} \mathrm{Kr}$ gas enclosed in a stainless steel cylinder were performed at Los Alamos National Laboratory (LANL) using the Detector for Advanced Neutron Capture Experiments (DANCE). ${ }^{85} \mathrm{Kr}$ is radioactive isotope with a half life of 10.8 years. As this was a low-enrichment sample, the main contaminants, the stable krypton isotopes ${ }^{83} \mathrm{Kr}$ and ${ }^{86} \mathrm{Kr}$, were also investigated. The material was highly enriched and contained in pressurized stainless steel spheres.
\end{abstract}

\section{Introduction}

Most of the elements with proton numbers higher than iron are produced through neutron-induced processes (neutron captures). There are two major processes, the rapid neutron-capture process ( $\mathrm{r}-$ process) and the slow neutron capture process (s-process) [4]. Only a few isotopes on the proton-rich side of the valley of stability get significant contributions from other processes.

If the conditions in the star make the rates for neutron capture comparable to the rate of $\beta$-decay for a particular isotope, the s-process path branches at that isotope: a fraction of the isotope transforms via neutron capture, while the other fraction $\beta$-decays. The branching ratio, or relative likelihood, for the different reactions depends on the physical conditions in the interior of the star - temperature, neutron density and electron density. At higher neutron densities with all other conditions equal, more nuclei of a given isotope capture a neutron before having the chance to $\beta$-decay. Thus, the branching ratios deduced from the isotopic ratios observed in stellar material provide the tools to effectively constrain modern models of the stars where the nucleosynthesis occurs. Therefore the fundamental rates, hence cross sections, for neutron capture and $\beta$-decay are essential.

A particularly interesting branching of the s-process occurs in the region around ${ }^{85} \mathrm{Kr}$ (Figure 1) [5]. The main path is indicated by green arrows while the red arrows show the path if ${ }^{85} \mathrm{Kr}$ captures a

^e-mail: fiebiger@iap.uni-frankfurt.de 


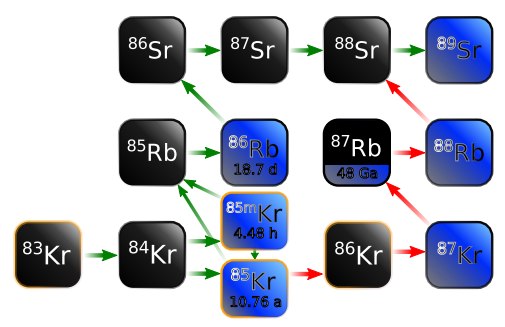

Figure 1. Reaction network during s-process nucleosynthesis around the important branch point ${ }^{85} \mathrm{Kr}$.

neutron. There is isomeric state with half life of $4.48 \mathrm{~h}$ which always decays before neutron capture either through $\beta$-decay or through internal conversion to the ground state.

\section{Samples}

As $\mathrm{Kr}$ is gaseous and in the case of ${ }^{85} \mathrm{Kr}$ radioactive, preparing suitable samples for the DANCE setup proved to be a major challenge. Two types of samples were designed for ${ }^{85} \mathrm{Kr}$ and ${ }^{83,86} \mathrm{Kr}$ respectively (Figure 2). Both had to fit in the existing beam pipe while simultaneously minimizing exposure of the sample holders. The first sample was a reactor produced ${ }^{85} \mathrm{Kr}$ sample containing fractions of ${ }^{83,84,85,86} \mathrm{Kr}$. Except for ${ }^{83} \mathrm{Kr}$, which has a higher Q-value $(10.52 \mathrm{MeV})$ than ${ }^{85} \mathrm{Kr}(9.86 \mathrm{MeV})$, all other Q-values are smaller than for ${ }^{85} \mathrm{Kr}$ which is important to distinguish between the isotopes. The gas is enclosed in a stainless steel cylinder with a height of $70 \mathrm{~mm}$ and a diameter of $3.7 \mathrm{~mm}$. The pressure inside the sample is about 0.05 bar (Figure 2, left).
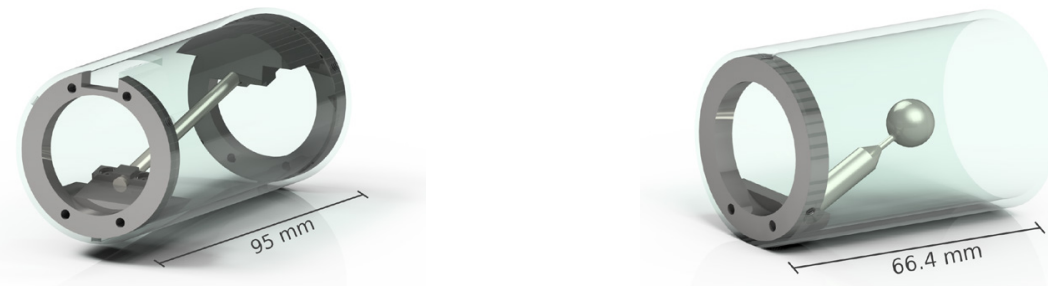

Figure 2. Left: Target holder for ${ }^{85} \mathrm{Kr}$. Right: Target holder for ${ }^{83} \mathrm{Kr} /{ }^{86} \mathrm{Kr}$.

Both the ${ }^{83} \mathrm{Kr}$ and ${ }^{86} \mathrm{Kr}$ samples were pressurized gas spheres with a diameter of $10.6 \mathrm{~mm}$ that were prepared with a special gas filling system at Goethe University Frankfurt. Gas pressure was 11 bar for the ${ }^{83} \mathrm{Kr}$ sample and 200 bar for the ${ }^{86} \mathrm{Kr}$ sample. This corresponds to a total mass of $0.02 \mathrm{~g} /$ $0.45 \mathrm{~g}$ respectively (Figure 2, right).

\section{Experiment Analysis}

The neutron capture cross sections are investigated using the time of flight technique. The analysis consists of several steps. As the gaseous samples were inside their respective containers, separate 
target holder measurements were also performed to characterize their contributions to the background. Furthermore corrections include pile-up and neutron scattering. A gold sample was used to determine the neutron flux.
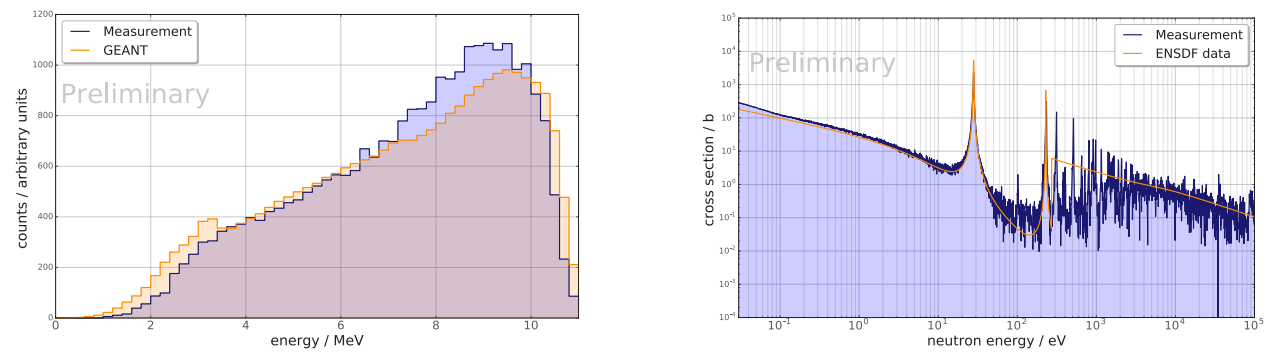

Figure 3. Left: Comparison of Esum spectrum of Cluster Multiplicity 4 with DICEBOX/GEANT and measurement. Right: Preliminary ${ }^{83} \mathrm{Kr}$ cross section.

Extensive DICEBOX/GEANT [6, 7] simulations were performed to reliably estimate the detector efficiency. The DICEBOX code was used to generate $\gamma$-ray cascades within the statistical model using known decay pattern below 3.55 MeV and models of $\gamma$-strength functions and nuclear level density at higher excitation energies. The resulting $\gamma$-cascades are than utilized in GEANT to simulation the response of the complete DANCE setup. A comparison of simulated and measured spectra is shown in Figure 3 (left). The difference between both spectra might be due to the fact that the utilized DICEBOX model does not fully describe the target nucleus.

As the purest sample, ${ }^{83} \mathrm{Kr}$ exhibits very little background from other isotopes. The preliminary cross section, shown in Figure 3 (right), is in very good agreement with recommended ENDF/B-VII.1 data [8]. Determination of the cross sections of the other isotopes is currently in progress.

\section{Acknowledgments}

This project was supported by the European Research Council under the European Union's Seventh Framework Programme (FP/2007-2013)/ERC Grant Agreement n. 615126. Milan Krtička acknowledges the support by Grant No. 13-07117S of the Czech Science Foundation.

\section{References}

[1] C. Abia et al., Astrophysical Journal, 559:1117 (2001)

[2] R. Raut et al. Cross-Section Measurements of the $86 \mathrm{Kr}(\mathrm{g}, \mathrm{n})$ Reaction to Probe the s-Process Branching at $85 \mathrm{Kr}$ (2013)

[3] Z. Y. Bao et al. Atomic Data Nucl. Data Tables, 76:70 (2000)

[4] R. Reifarth et al. Journal of Physics G Nuclear Physics, 41(5):053101 (2014)

[5] C. Abia et al. Astrophysical Journal, 559:1117 (2001)

[6] F. Bečvář, Nucl. Instr. Meth. A, 417:434 (1998)

[7] J. Apostolakis, CERN program library long writeup, W5013 (1993)

[8] M. B. Chadwick et al., Nuclear Data Sheets, 112:2887 (2011) 
